\title{
Evaluation of a novel biologically inspired Trajectory Generator in Human-Robot Interaction
}

\author{
Markus Huber ${ }^{1}$, Helmuth Radrich ${ }^{2}$, Cornelia Wendt ${ }^{3}$ Markus Rickert $^{2}$, \\ Alois Knoll ${ }^{2}$, Thomas Brandt ${ }^{4}$, Stefan Glasauer ${ }^{1}$ \\ ${ }^{1}$ Center for Sensorimotor Research, Institute of Clinical Neurosciences, Ludwig-Maximilians-Universität München \\ ${ }^{2}$ Robotics and Embedded Systems Lab, Department of Informatics, Technische Universität München \\ ${ }^{3}$ Human Factors Institute, Universität der Bundeswehr München \\ ${ }^{4}$ Chair of Clinical Neurosciences, Ludwig-Maximilians-Universität München
}

\begin{abstract}
In many future joint-action scenarios, humans and robots will have to interact physically in order to cooperate successfully. Ideally, human-robot interaction should not require training on the human side, but should be intuitive and simple. Previously, we reported on a simple case of physical human-robot interaction, a hand-over task [1]. Even such a basic task as manually handing over an object from one agent to another requires that both partners agree upon certain basic prerequisites and boundary conditions. While some of them are negotiated explicitly, e.g. by verbal communication, others are determined indirectly and adaptively in the course of the cooperation. In the previous study we compared a humanhuman hand-over interaction with the same task performed by a human and a robot. However, the trajectories used for the robot, a conventional trapezoidal velocity profile in joint coordinates and a minimum-jerk profile of the end-effector, have little resemblance to the natural movements of humans. In this study we introduce a novel trajectory generator that is a variation of the traditional minimum-jerk profile, the 'decoupled minimum-jerk' profile. Its trajectory is much closer to those observed in human-human experiments. We evaluated its performance concerning human comfort and acceptance in a simple hand-over experiment by using a post-test questionnaire. The evaluation of the questionnaire revealed no difference with respect to comfort, human-likeness, or subjective safety of the new planner compared to the minimum-jerk profile. Thus, the 'decoupled minimum-jerk' planner, which offers important advantages with respect to target approach, proved to be a promising alternative to the previously used minimum-jerk profile.
\end{abstract}

\section{INTRODUCTION}

Robots have been employed successfully in industrial settings for improving productivity and for performing dangerous tasks. Due to the recent remarkable improvements in robotic intelligence and technology, it is expected that robots will soon coexist with humans and assist them in joint action tasks. To this end, future robots must be able to cooperate with humans in a safe and user-friendly manner. This implies potentially dangerous physical contact, e.g. in a situation where a robot is assisting a human in a construction task. This kind of interaction can be compared to the relation between a master craftsman and his apprentice, and it is only efficient and safe if the apprentice knows what the master wants to do next, so that he can act accordingly. If we want a robot to serve as an efficient apprentice, it needs to move and react just in time and without alarming his master by unexpected actions or by movements that are perceived as being too fast or too unfamiliar. In other words, efficient human-robot interaction requires understanding the other's actions and intentions [2].

One of the strategies in the field of robotics is to learn from human-human interaction and to transfer the results to technical systems [3]. Human single-handed actions, general movements and pointing or reaching, have already been well-studied and various mathematical models have been proposed to describe their kinematics (for review see [4]). In general, those algorithms are based on a certain optimization criterion, e.g. minimum-jerk [5] or minimum-variance [6]. Studies of the kinematics of grasping similarly revealed characteristic patterns of behavior [7], [8], [9]. Some of these results have already been implemented in robotic systems to simulate human behavior [10]. However, studies about cooperative strategies in humans, specifically concerning manual joint-action, are relatively new. There are only few studies concerning e.g. action patterns for competitive and cooperative behaviors [11], the transfer of objects in jointaction [12], or cooperative lifting of objects [13]. And extending these results to the field of human-robot interaction raises completely new questions, e.g. about the humans' acceptance of the robot and the efficiency of the interaction. Latest results in this field are reported in [14], [15].

For human robot interaction, it might be advantageous to use human inspired motor control theories to simulate human-like robot movements. We humans are used to velocity profiles and trajectories similar to our own motor systems from the experiences gained interacting with other individuals throughout our lives. It therefore sounds reasonable, that humans are going to feel more comfortable when interacting with a robot using natural, human-like movements. The first theory describing human motor control and explaining the underlying principle was the minimum jerk theory introduced by [5]. Several other theories were developed later, using other minimum principles to improve human data description [16] [6]. Most current minimum principles in motor control have been chosen empirically, based on their success at predicting the characteristics of 
arm movement, and biological relevance has often only been considered as an afterthought [4].

In the present work we introduce a new trajectory generator that is a variation of the traditional minimum-jerk profile. We call it 'decoupled minimum-jerk' since it treats the $\mathrm{X}$ $\mathrm{Y}$ and $\mathrm{Z}$ components of the 3D-trajectory separately and by doing this 'decouples' the height component of the movement from the planar component. This leads to a much more human-like trajectory. The decoupled minimum-jerk profile is compared to the profiles previously used (a conventional trapezoidal velocity profile in joint coordinates [17] and a minimum-jerk profile of the end-effector [5]), by means of a psychological questionnaire designed to evaluate the humans subjective impression of interaction safety, human-likeness and acceptance of the robots movements.

In our experiment six wooden cubes are handed over by a humanoid robot (apprentice) to the human subject (master) (Fig. 5) using the three different movement trajectories (trapezoid, minimum-jerk, decoupled minimum-jerk). Important parameters of the robots motion, like timing, duration or hand-over position, were derived from analogous human-human experiments. The human participants' subjective impressions regarding interaction safety, as well as human-likeness and acceptance of the robot's movements were measured by means of a psychological questionnaire.

\section{HUMAN-HUMAN HAND-OVER EXPERIMENTS}

\section{A. Experimental Setup}

We measured hand movements in human subjects during a hand-over task using the magnet-field based motion tracking system Polhemus Liberty. The two test subjects were sitting opposite to each other at a table (width $75 \mathrm{~cm}$ ) (Fig. 1). The hand positions of the subjects were recorded by the tracking system. Tracking sensors were placed on the back of the subject's hands and shoulders. Six wooden cubes $(3 \times 3 \times$ $3 \mathrm{~cm}$ ) were handed over by one subject (giving subject) to the other (receiving subject). The size of the cubes required a precision grip. The cubes were placed in one row on predefined locations on the table. Corresponding locations on the other side of the table served as targets for placing the cubes after each hand-over. The distance between the two rows of cube locations was $50 \mathrm{~cm} .32$ test subjects distributed in 16 pairs participated in the experiment. The subjects were instructed before the start of the experiment as follows: 'The person with the cubes will hand over the cubes, one after the other. The start of each handing over will be indicated by a sound in your headphones. The other person should place them on the marks in front of him.' The trigger sound could be heard only by the giving subject (head-phones) and was introduced to the experimental setting in order to avoid the adaptation of the subjects to a fixed timing pattern. The waiting period in between two consecutive hand-overs was randomly chosen between 4.8 and 6 seconds.

\section{B. Human-Human Results}

In the following we will focus on the average humanhuman interactions end-effectors (hand) trajectories and ve-

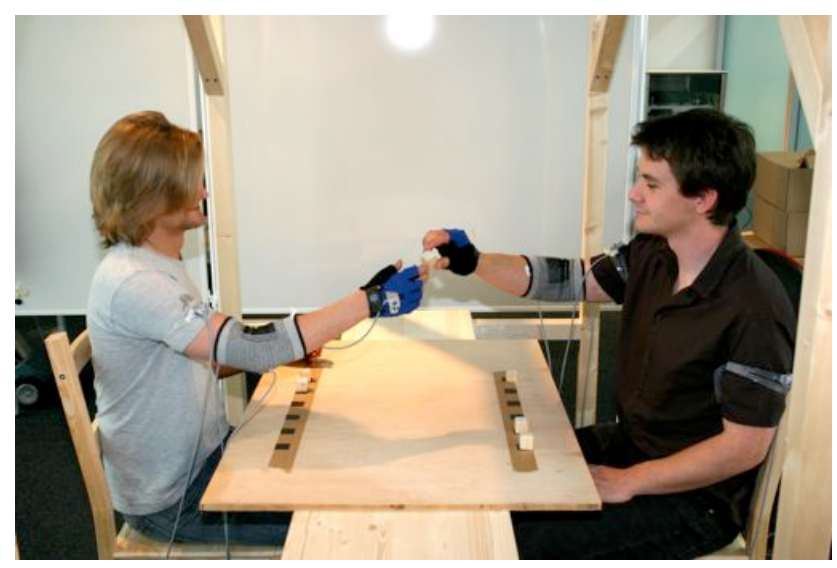

Fig. 1. Human-Human handover experiment.

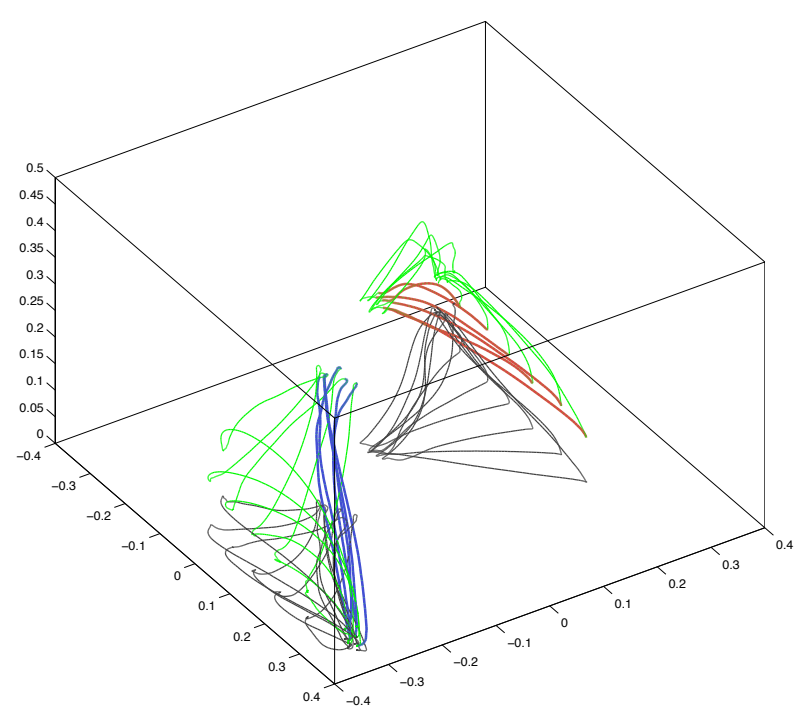

Fig. 2. Trajectories of a human-human hand-over with 6 cubes. The red curve shows the movements from the initial cube-positions to the handover position for the giving subject. The receiving subjects movement is plotted in blue. The complete trajectories of the two subjects are plotted in light green, showing the placing of the cubes and the movement back to the resting position. The projection of the trajectories onto the XY-plane is displayed in gray.

locity profiles. The typical trajectories of a pair of subjects during the hand-over task is shown in Fig. 2. The giving subject in Fig. 2 is plotted on the right side; his movement from grasping a cube to the hand-over position is colored blue. The movement from the handing over position back to the initial position is plotted in light green, as well as the movement from the initial position to one of the six cubes. Like in other works [18], it is immediately obvious that the trajectories of both, the giving and the receiving subjects are curved in space instead of being straight lines.

\section{A Minimum Jerk Variant: Decoupled Minimum Jerk}

As interactions between robots and humans take place in three-dimensional space, it is necessary to transfer motor 
control principles into three dimensions. It is reasonable to start with the most simple motor control theory, the minimum jerk principle. The big advantage of this basic approach is that it is fully analytically solvable.

Traditionally, the minimum jerk theory in two dimensions leads to the objective function $\mathrm{c}(\mathbf{r})$ (1), where $\mathbf{r}$ is the end effector positions-vector and $t_{e}$ is the duration of the movement (assumed the movements starts at $t_{0}=0$,).

$$
c(\mathbf{r})=\frac{1}{2} \int_{0}^{t_{e}}\left|\frac{d^{3} \mathbf{r}}{d t^{3}}\right|^{2} d t
$$

Minimizing this objective function leads to a fifth-order polynomial. Given initial/end position, velocity and acceleration for the trajectory, we can specify the polynomial coefficients (2). The first derivative of this equation results in the positions velocity profile (3), where $\mathbf{r}_{0}$ and $\mathbf{r}_{e}$ denote the initial and end-positions of the end effector, with the desired duration $t_{e}$.

$$
\begin{gathered}
\mathbf{r}(t)=\mathbf{r}_{0}+\left(\mathbf{r}_{0}-\mathbf{r}_{e}\right)\left(15\left(\frac{t}{t_{e}}\right)^{4}-6\left(\frac{t}{t_{e}}\right)^{5}-10\left(\frac{t}{t_{e}}\right)^{3}\right) \\
\dot{\mathbf{r}}(t)=\left(\mathbf{r}_{0}-\mathbf{r}_{e}\right)\left(60 \frac{t^{3}}{t_{e}^{4}}-30 \frac{t^{4}}{t_{e}^{5}}-30 \frac{t^{2}}{t_{e}^{3}}\right)
\end{gathered}
$$

If we specify $\dot{\mathbf{r}}$ as a 3 dimensional vector, these equations describe minimum jerk trajectories generalized to three dimensions. Like widely know, equation 2 expresses the position of the end-effector at a given time and describes as a straight line. This however does not fit the observed human trajectories in hand-over experiments, which are curved in space (see Fig 2).

Figure 3 shows the averaged velocity profiles from 16 human subjects for the z-component, the absolute velocity, and the horizontal XY-components of the velocity. The top displays the giving, the bottom the receiving subjects. Figure 4 shows the averaged trajectories of the subjects (height vs. horizontal XY-plane, plotted in blue). Both profiles exhibit a steep rising in the beginning and a shallow descent at the end of the movement. The human velocity profiles 3 show a steep ascent in the beginning and a shallow descent at the end of the movement. The symmetric minimum jerk fits (dotted lines for each component) do not fit this trajectories well, even though the receiving subject is fitted better than the giving subject.

The different shapes of the velocity profiles of the giving and receiving subjects lead to the assumption, that the velocity profile is task dependent. The givings subjects task is to present the item at a comfortable position in a way that it can be easily grasped. This requires a slow approach to the handover position, so that the receiving subject is able to estimate this location accurately and is able to start planing his movement before the giving subject reaches the endpoint. In contrast, the receiving subjects task is to just move to the hand-over position and grasp the item, which leads to a more bell-shaped velocity profile.
TABLE I

TIMES FOR REACHING THE SINGLE COMPONENTS OF THE THREE DIMENSIONAL END-POINT OF THE MOVEMENT DURING HUMAN-HUMAN EXPERIMENTS.

\begin{tabular}{|c|c|c|c|c|}
\hline Subject & $t_{e x}$ & $t_{e y}$ & $t_{e z}$ & ratio \\
\hline Giving & $1.04 \mathrm{sec}$ & $1.04 \mathrm{sec}$ & $0.76 \mathrm{sec}$ & 1.37 \\
\hline Receiving & $0.86 \mathrm{sec}$ & $0.86 \mathrm{sec}$ & $0.61 \mathrm{sec}$ & 1.41 \\
\hline
\end{tabular}

In contrast to the minimum jerk model in three dimensional coordinates, the combination of the independent minimum jerk fits for the horizontal XY-plane and the Zcomponent lead to an asymmetric total velocity profile (dotted light green lines in Figure 4 and 3), which significantly better fits the measured velocity profile of the receiving human compared to the minimum jerk fit (dotted dark green line). This independent consideration of the height and horizontal components lead to a curved trajectory in space (shown in Fig 4 as dotted red line). Here again, the receiving subjects' averaged trajectories are fitted better than the giving subjects' .

Equation 4 describes the decoupled minimum jerk movement trajectory. The end times $t_{e x y}$ and $t_{e y}$ from the regular three dimensional minimum jerk trajectory are set equal and are expressed by $t_{e x y}$ here.

$$
\dot{\mathbf{r}}(t)=\left(\mathbf{r}_{0}-\mathbf{r}_{e}\right)\left(\begin{array}{c}
60 \frac{t^{3}}{t_{e x y}^{4}}-30 \frac{t^{4}}{t_{e x y}^{5}}-30 \frac{t^{2}}{t_{e x y}^{3}} \\
60 \frac{t^{3}}{t_{e x y}^{4}}-30 \frac{t^{4}}{t_{e x y}^{5}}-30 \frac{t^{2}}{t_{e x y}^{3}} \\
60 \frac{t^{3}}{t_{e z}^{4}}-30 \frac{t^{4}}{t_{e z}^{5}}-30 \frac{t^{2}}{t_{e z}^{3}}
\end{array}\right)
$$

Using the different durations for reaching the $\mathrm{Z}$ and the horizontal XY-end-points extracted from the human-human experiments (Table I) we get trajectories that are much closer to averaged human trajectories than a generalized minimum jerk model. In Figure 4 we also plotted the resulting trajectories from the minimum jerk model (dotted green line) and the trajectories calculated with our new model, in addition to the averaged human trajectories.

\section{HuMAN-Robot HAND-OVER EXPERIMENTS}

In this work we were interested to see whether humans would recognize significant (subjective) differences during our human-robot cube had-over experiment in between the three different end-effector trajectories. We could demonstrate already in our previous work [1] that there is a significant difference regarding the human reaction times for different velocity profiles. The question was now if the human subjects would also feel a subjective difference concerning their safety, comfort and the naturalness of the robots movements.

\section{A. Experimental Setup}

The humanoid robot system JAST - described in detail in [19] - was employed for the handover experiment. The JAST system consists of two industrial Mitsubishi robot arms attached to a torso simulating a human upper body. The 

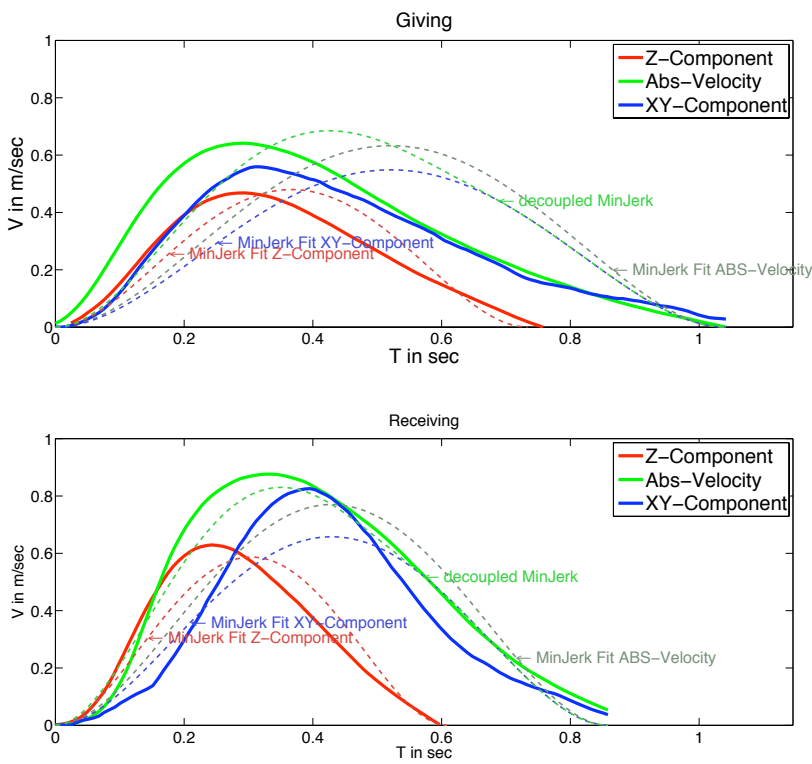

Fig. 3. Averaged velocity profile of the giving (top) and receiving (bottom) subject. The red curve is the velocity of the Z-component, the green cuve is the absolute velocity and the blue curve is the abolute velocity for the horizontal XY- components. The minimum jerk fit is plotted as dotted line. It can be seen, that the superposition of the xy-comonent and the z-component leads to a better fit than the fit of the abs-velocity.
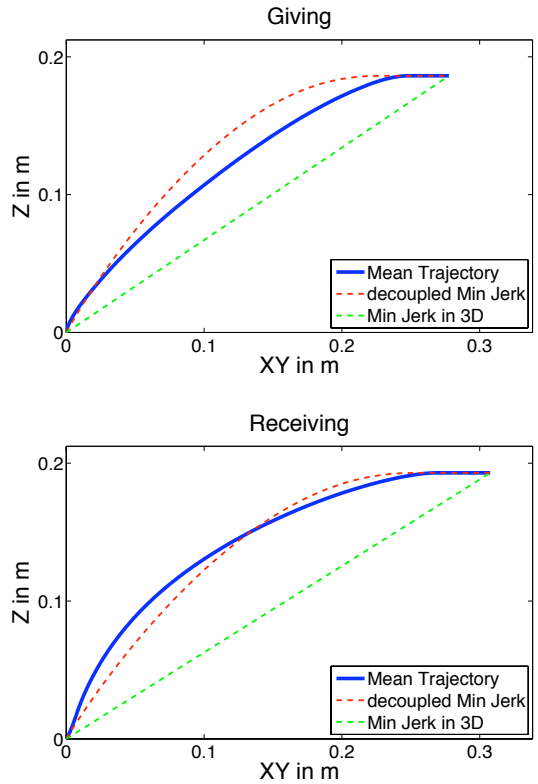

Fig. 4. Averaged trajectories of the giving and receiving movements (blue). The XY-plane is reduced to one dimension. The dotted green curve is the minimum jerk fint the red curve is the decoupled minimum jerk fit. grippers are equipped with force torque sensors. On the top of the torso rests an Phillips ICat expressive head. However, it was covered during our experiments to prevent any distraction from the arm movements. In addition, subjects were instructed to carefully focus on the trajectories. We only used the systems right arm for the handover.

The Setup of the experiment was similar to the humanhuman experiment [20] concerning initial and target position of the cubes, as well as the handover position and duration. We investigated a handing over task of 6 cubes for each trajectory generator. Figure 5 shows the JAST robot system in such a hand-over situation. In contrast to the human-human experiment, it was not possible to lay down the gripper on the table before starting the task. Instead, the robot's starting position was set at the same position but $19 \mathrm{~cm}$ above the table. Starting from that position in the air, the robot arm would pick up a cube and move it to the predetermined handover position. The grippers were programmed to open as soon as the force torque sensors detected that someone was grasping the cube.

A total of 30 subjects were tracked with three different trajectory planners consecutively: (1) the trapezoidal velocity in joint coordinates, (2) the minimum jerk, and (3) the decoupled minimum jerk trajectory planner (see figure 6). The duration of the robot's movements were identical for all trajectories so that it took the same time for the robot to reach the end position in each trajectory condition. After each single handover, the robot moved back to its resting position and waited for a duration varying from 4.8 to 6.0 seconds, so that subjects could not adapt themselves to a periodic timing.

The sequence of the three different trajectories was permutated so that every variant was used for the same number of subjects. We briefed the subjects to act naturally and to focus their attention on the trajectory of the robot arm. After each profile, subjects were asked...

- how secure they felt during the interaction,

- how hardly the robot's movement was predictable

- how humanlike the robot's movements had looked,

- how comfortable the interaction had been, and

- how abrupt the beginning of the robot's movement had appeared.

Each question was rated on a Likert scale ranging from 1 ("'not at all"') to 8 ("'absolutely"').

\section{B. Human-Robot Results}

During the course of the experiment, we noticed that the trapezoidal velocity profile had significant disadvantages when grasping the cubes directly and not through a via point. That way, trajectories ran very close to the table surface, especially shortly after grasping the cubes. For other scenarios with randomly distributed items, this might result in hitting nearby items. Furthermore, the velocity of the trapezoidal profile is a discontinuous function that leads to observable vibrations during and at the end of the movements. This also leads to problems when trying to determine if there is 

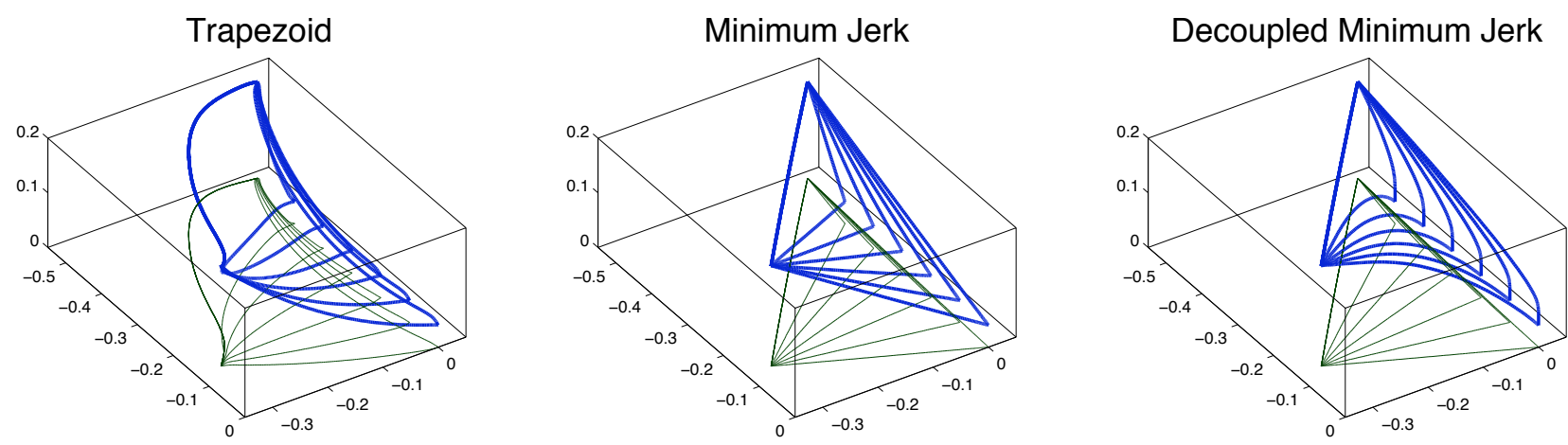

Fig. 6. Robot trajectories for the hand-over task. From left to right: trapezoidal joint velocity, three dimensional minimum jerk, decoupled minimum jerk. The resting position is located in the upper corner for all trajectories. From there, the gripper is moving to the different cube positions. After picking one up, the gripper is lifting it to the hand-over position located near the lower corner of the plots. After the hand-over, the gripper is moving back to the resting position

TABLE II

RESUlts OF THE FRIEDMAN's TEST FOR THE RATINGS OF FIVE DIFFERENT INTERACTION ASPECTS REGARDING THE THREE MOVEMENT PROFILES

\begin{tabular}{|c|c|c|c|c|c|c|}
\hline & $T_{\text {mean }}$ & $M_{\text {mean }}($ rank $)$ & $D_{\text {mean }}($ rank $)$ & chi -2 & $d f$ & Sign. \\
\hline 3) The robot moved human-like. & $4.93(1.77)$ & $5.43(2.00)$ & $5.67(2.23)$ & 3.960 & 2 & .138 \\
\hline 5) The beginning of the robot's movement was abrupt. & $3.57(1.95)$ & $3.20(1.73)$ & $4.10(2.32)$ & 6.589 & 2 & $.037 *$ \\
\hline
\end{tabular}

Mean values and mean ranks for the 3 velocity profiles trapeziodal (T), minimum jerk (M), and decoupled minimum jerk (D), as well as results from the nonparametric Friedman Test for repeated measures ( $\mathrm{N}=30$ participants). A p-value smaller than .01 (**) or .05 (*) indicates statistically significant differences between at least 2 of the 3 profiles.

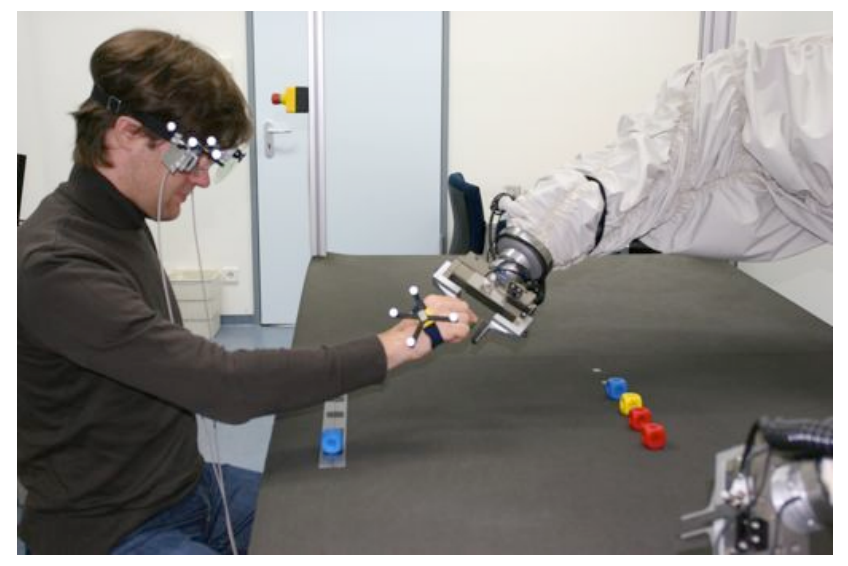

Fig. 5. Picture of the experimental JAST-Human handover setting.

an external force (elicited by the human grasping the cube) which triggered the gripper to open.

The analysis of the questionnaire revealed that all in all, the trajectories were rated very similar. As shown in Table (II), participants evaluated feelings of high security (mean value 7.2 to 7.4 ) and high predictability (2.8-3.1, negatively poled) for all profiles. Based on the mean values, the decoupled minjerk profile was rated most human-like (item 3), followed by the minimum jerk and the trapezoidal profile. Yet, these differences did not yield significant results.
In contrast, differences between the different trajectories could be found for comfort during interaction (item 4) and abruptness of the robot's movements (item 5). Post-hoc Tests (Wilcoxon) showed that interaction was rated significantly more comfortable for the minimum jerk $(\mathrm{Z}=-2.298, \mathrm{p}=.022$, based on negative ranks), as well as for the decoupled minimum jerk $(\mathrm{Z}=-2.44, \mathrm{p}=.015$, based on negative ranks) compared to the trapezoidal profile. With regard to abrupt robot movements, only the comparison of minimum jerk and decoupled minimum jerk yielded significant results $(\mathrm{Z}=-$ 2.382, $\mathrm{p}=.017$, based on negative ranks). At first glance, it is surprising that humans rated the decoupled minjerk profile as more abrupt as the others (as indicated by the means, see Table II). It might be that test subjects confused a sudden initiation of the movement with the maximum velocity, which was indeed the highest for the decoupled minjerk profile. A very interesting result is the significant correlation between item 3 (human-like movement) and item 4 (comfortabel interaction) for all three trajectories (T-profile: $\mathrm{r}=.669, \mathrm{p}=.000$; M-profile: $\mathrm{r}=.640 . \mathrm{p}=.000$; D-profile: $\mathrm{r}=.433$, $\mathrm{p}=.017$ ). This underlines the assumption made in the introduction that human-like robot movements are experienced as more comfortable by humans.

\section{Discussion}

In this paper we investigated human hand trajectories in a typical cooperative interaction, a hand-over task. We found 
that human hand trajectories are curved in space and that they are not properly described by the generalized three dimensional minimum jerk trajectory. The shapes of the velocity profiles are different for the giving and the receiving subjects. We assume that the shapes of the velocity profile depend on the different goals (giving or receiving), and are therefore task dependent.

We presented a novel trajectory generator that better resembles human trajectories, based on the minimum jerk trajectory but with a decoupling of the movements height (Z) component. The suggested 'decoupled minimum jerk' trajectory generator better fits the curved nature of human trajectories. The decoupled minimum jerk trajectory was compared to the standard trapezoid and to the three dimensional minimum jerk profile.

Obviously, the trapezoid velocity profile is not a good choice for human-robot interactions because of its discontinuous velocity function and the resulting vibrations during and after the movement, that not only disturb the force torque sensor used to detect the grip on the cube but are also irritating to the human subjects. The use of the trapezoid profile also results in trajectories close to the tables surface in the vicinity of the target cubes, which hinders its (direct) use in scenarios with densely packed objects on the table. This is also true, though to a lesser extent, for the three dimensional minimum jerk profile. Here, the decoupled minimum jerk trajectory generator performed best. It generates the trajectory that most closely resembles the human data. It also approaches the cubes from above (like humans do) so that it can be directly used in the beforementioned scenarios, with many objects on the table or with objects lying close to each other, without any modifications.

The individual evaluation of the asked questions showed high values in the safety and comfort ranking for all profiles. The decoupled minimum jerk profile was rated most humanlike, even though the difference was not significant.

The joint evaluation of the whole questionnaire showed that our hypothesis that a human-like movement leads to a better overall acceptance, is right.

The next step for the continuation of our work in humanrobot interaction will be the implementation of the HoffArbib next step planer [21] into our decoupled minimum jerk trajectory generator. This will allow an online change of the trajectories endpoints while keeping the movement smooth.

\section{ACKNOWLEDGEMENT}

This work is supported by the DFG cluster of excellence "CoTeSys" (www. cotesys.org) and by the EU FP6 IST Cognitive Systems Integrated Project "JAST" (FP6-003747IP) (www. euprojects-jast. net).

\section{REFERENCES}

[1] M. Huber, M. Rickert, A. Knoll, T. Brandt, and S. Glasauer, "Humanrobot interaction in handing-over tasks," in Robot and Human Interactive Communication, 2008. RO-MAN 2008. The 17th IEEE International Symposium on Robot and Human Interactive Communication. München, Germany: IEEE Robotic and Automation Society, aug 2008, pp. 107-112.
[2] U. Castiello, "Understanding other people's actions: Intention and attention," Journal of Experimental Psychology-Human Perception and Performance, vol. 29, no. 2, pp. 416-430, Apr 2003.

[3] W. Erlhagen, A. Mukovskiy, and E. Bicho, "A dynamic model for action understanding and goal-directed imitation," Brain Research, vol. 1083, pp. 174-188, Apr 2006.

[4] S. E. Engelbrecht, "Minimum principles in motor control," Journal of Mathematical Psychology, vol. 45, no. 3, pp. 497-542, Jun 2001.

[5] T. Flash and N. Hogan, "The coordination of arm movements - an experimentally confirmed mathematical-model," Journal of Neuroscience, vol. 5, no. 7, pp. 1688-1703, 1985.

[6] C. M. Harris and D. M. Wolpert, "Signal-dependent noise determines motor planning," Nature, vol. 394, no. 6695, pp. 780-784, Aug 1998.

[7] M. Jeannerod, "Intersegmental coordination during reaching at natural visual objects," in Attention and performance IX. Lawrence Erlbaum, 1981, pp. 153-169.

[8] - "The timing of natural prehension movements," Journal of Motor Behaviour, no. 16, pp. 235-254, 1984.

[9] J. B. J. Smeets and E. Brenner, "A new view on grasping," Motor Control, vol. 3, no. 3, pp. 237-271, Jul 1999.

[10] G. Simmons and Y. Demiris, "Object grasping using the minimum variance model," Biological Cybernetics, vol. 94, no. 5, pp. 393-407, May 2006.

[11] I. Georgiou, C. Becchio, S. Glover, and U. Castiello, "Different action patterns for cooperative and competitive behaviour," Cognition, vol. 102, no. 3, pp. 415-433, Mar 2007.

[12] R. G. J. Meulenbroek, J. Bosga, M. Hulstijn, and S. Miedl, "Jointaction coordination in transferring objects," Experimental Brain Research, vol. 180, no. 2, pp. 333-343, Jun 2007.

[13] J. Bosga and R. G. J. Meulenbroek, "Joint-action coordination of redundant force contributions in a virtual lifting task," Motor Control, vol. 11, no. 3, pp. 235-258, Jul 2007.

[14] S. Shibata, T. Yamamoto, M. Jindai, and A. Shimizu, "A fundamental approach of avoidance planning of robots considering human emotions," JSME International Journal Series C-Mechanical Systems Machine Eelements and Manufacturing, vol. 46, no. 1, pp. 270-277, Mar 2003.

[15] S. Shibata and H. Inooka, "Psychological evaluations of robot motions," International Journal of Industrial Ergonomics, vol. 21, no. 6, pp. 483-494, Jun 1998.

[16] Y. Uno, M. Kawato, and R. Suzuki, "Formation and control of optimal trajectory in human multijoint arm movement - minimum torquechange model," Biological Cybernetics, vol. 61, no. 2, pp. 89-101, 1989.

[17] J. J. Craig, Introduction to Robotics: Mechanics and Control, 3rd ed. Upper Saddle River, NJ: Pearson Prentice Hall, 2005.

[18] M. D. K. Breteler, R. G. J. Meulenbroek, and S. C. A. M. Gielen, "Geometric features of workspace and joint-space paths of $3 \mathrm{~d}$ reaching movements," Acta Psychologica, vol. 100, no. 1-2, pp. 37-53, Nov 1998.

[19] M. Rickert, M. E. Foster, M. Giuliani, T. By, G. Panin, and A. Knoll, "Integrating language, vision and action for human robot dialog systems," in Proceedings of HCI International 2007, 2007, pp. 987995.

[20] M. Huber, A. Knoll, T. Brandt, and S. Glasauer, "Handing-over a cube: spatial features of physical joint action," Annals of the New York Academy of Sciences, vol. 1431, 2008, (in press).

[21] B. Hoff and M. A. Arbib, "A model of the effects of speed, accuracy, and perturbation on visually guided reaching," in Control of Arm Movement in Space: Neurophysiological and Computational Approaches, ser. Experimental Brain Research, R. Caminiti and et. al., Eds. Springer-Verlag, 2002, pp. 285-306. 